\title{
Postinfarct Intracardiac Mass with Severe Mitral Regurgitation: Late Rupture of One of the Heads of Posteriomedial Papillary Muscle
}

\author{
Prashanth Panduranga
}

\begin{abstract}
Acute ischemic mitral regurgitation is due to complete or partial rupture of either anterolateral or posteriomedial papillary muscles occurring within a week of acute myocardial infarction. Chronic ischemic mitral regurgitation is due to postinfarct remodeling leading to imbalance between tethering and closing forces of mitral valve apparatus. We present a 64-year-old male, presenting with acute pulmonary edema secondary to severe mitral regurgitation, a week after his myocardial infarction. Transthoracic echocardiogram detected a mobile intracardiac mass near anterior mitral leaflet with no clear-cut intracardiac origin of this mass. Perioperative transesophageal echocardiography detected rupture of one of the heads of posteriomedial papillary muscle. This case illustrates a rare presentation of postinfarct rupture of one of the heads of posteriomedial papillary muscle occurring late after infarction presenting as an intracardiac mass, which can have surgical implications.
\end{abstract}

Keywords: Mitral regurgitation, Intracardiac mass, Papillary muscle rupture, Transesophageal echocardiography.

How to cite this article: Panduranga P. Postinfarct Intracardiac Mass with Severe Mitral Regurgitation: Late Rupture of One of the Heads of Posteriomedial Papillary Muscle. J Perioper Echocardiogr 2014;2(1)29-31.

Source of support: Nil

Conflict of interest: None

\section{INTRODUCTION}

Acute ischemic mitral regurgitation is due to complete or partial rupture of either anterolateral or posteriomedial papillary muscles occurring within a week of acute myocardial infarction. Chronic ischemic mitral regurgitation is due to postinfarct remodeling leading to imbalance between tethering and closing forces of mitral valve apparatus. ${ }^{1-3}$ We present a 64 -year-old male, presenting with acute pulmonary edema secondary to severe mitral regurgitation, a week after his myocardial infarction.

Senior Specialist

Department of Cardiology, Royal Hospital, Muscat, Oman

Corresponding Author: Senior Specialist, Department of Cardiology, Royal Hospital, Muscat, Oman, Phone: 96892750349, e-mail: prashanthp_69@yahoo.co.in

\section{CASE REPORT}

A 64-year-old man with history of diabetes suffered an inferior ST-elevation myocardial infarction and was thrombolyzed in a regional hospital. There was no history of any valvular disease previously. A week after discharge, he presented with flash pulmonary edema without shock and detection of a new pan systolic murmur which was not documented previously. After ventilation and stabilization, a bedside transthoracic echocardiogram showed nondilated left ventricle with akinetic segments except for lateral wall with severe eccentric mitral regurgitation (MR) and an ejection fraction of $25 \%$. There was a mobile mass detected near anterior mitral leaflet with no clear-cut intracardiac origin of this mass with intact looking papillary muscles (PM). Urgent coronary angiogram showed tight lesions in the proximal left anterior descending artery, first diagonal artery and posterior descending arteries. His left circumflex system did not have significant lesions. He underwent immediate mitral valve (MV) repair and coronary artery bypass graft surgery. A perioperative transesophageal echocardiogram (TEE) done showed a mobile echogenic mass measuring $18 \mathrm{~mm}$ in length, seen behind the anterior mitral leaflet prolapsing into the left atrium as well as in the left ventricle (Figs 1A to C, arrowheads, Movie 1). There was no clear-cut intracardiac attachment of this mass. There was significant prolapse of P2 segment of posterior mitral leaflet (PML) without any flailing into the left atrium. The anterior mitral leaflet appeared normal with no prolapse. Annulus was not dilated. Even the major chordae to both the leaflets appeared intact. There was severe eccentric anteromedial directed MR (Fig. 2A, arrowheads). However, on transgastric view, the anterolateral PM head was single and appeared intact, but the posteriomedial PM head appeared bilobed with an abnormal cut-off of one of the heads of PM suggestive of PM rupture (Fig. 2B, arrowheads, Movie 2). There was hyper contractile lateral wall.

At surgery, MV was grossly incompetent due to $\mathrm{P} 2$ and P3 prolapse with one chordae to $\mathrm{P} 2$ ruptured along with one of the heads of posteriomedial PM. These chordae with the PM head were inserted into the remaining posterior PM with pericardial pledgetted sutures along with chordae 
transfer and insertion of a $28 \mathrm{~mm}$ Physio annuloplasty ring. Postoperatively, this mass was not seen.

\section{DISCUSSION}

This case brings in two important questions, namely, origin of the mobile intracardiac left-sided mass as well as exact mechanism of ischemic MR in this patient. The clinical scenario rules out common causes of a mobile intracardiac mass, like thrombus, vegetation or tumor. Ischemic MR is of two types: acute and chronic. ${ }^{1-3}$ Acute ischemic MR is secondary to papillary muscle infarction and rupture (either complete or partial rupture of PM head). ${ }^{1-3}$ Such patients usually present in cardiogenic shock due to inability of the left ventricle to accustom to acute volume overload. ${ }^{1}$ In case of PM rupture, echocardiographic characteristics described in the literature include a mobile mass attached to the chordae tendineae and to the MV, mobile echo density in the left ventricular body (not attached to the ventricular wall) with an erratic motion (out of phase with ventricular wall motion), a mobile mass prolapsing into the left atrium during systole and into left ventricle during diastole, erratic motion of the PM attached to the ventricular wall, an abnormal cutoff of one PM and lastly prominent MV cusp prolapse or flail MV leaflet. $^{4,5}$

Chronic ischemic MR is defined as MR occurring more than 1 week after myocardial infarction with: (1) one or more left ventricular segmental wall motion abnormalities; (2) significant coronary disease in the territory supplying the wall motion abnormality; and (3) structurally normal MV leaflets and chordae tendineae. In chronic ischemic MR, post-infarction remodeling leads to an imbalance between increased tethering forces (PM displacement, tethering of the leaflets/chordae and decreased leaflet coaptation) and reduced closing forces (reduced contractility, dyssynchrony of the papillary muscles, intra-left ventricular dyssynchrony, annular dilatation). ${ }^{1-3}$ There are two PMs anterolateral PMwhich has dual blood supply of left anterior descending artery and circumflex artery is a single large structure; whereas the posteromedial PM supplied by right coronary artery is known to have one to three heads. ${ }^{6}$ The two papillary

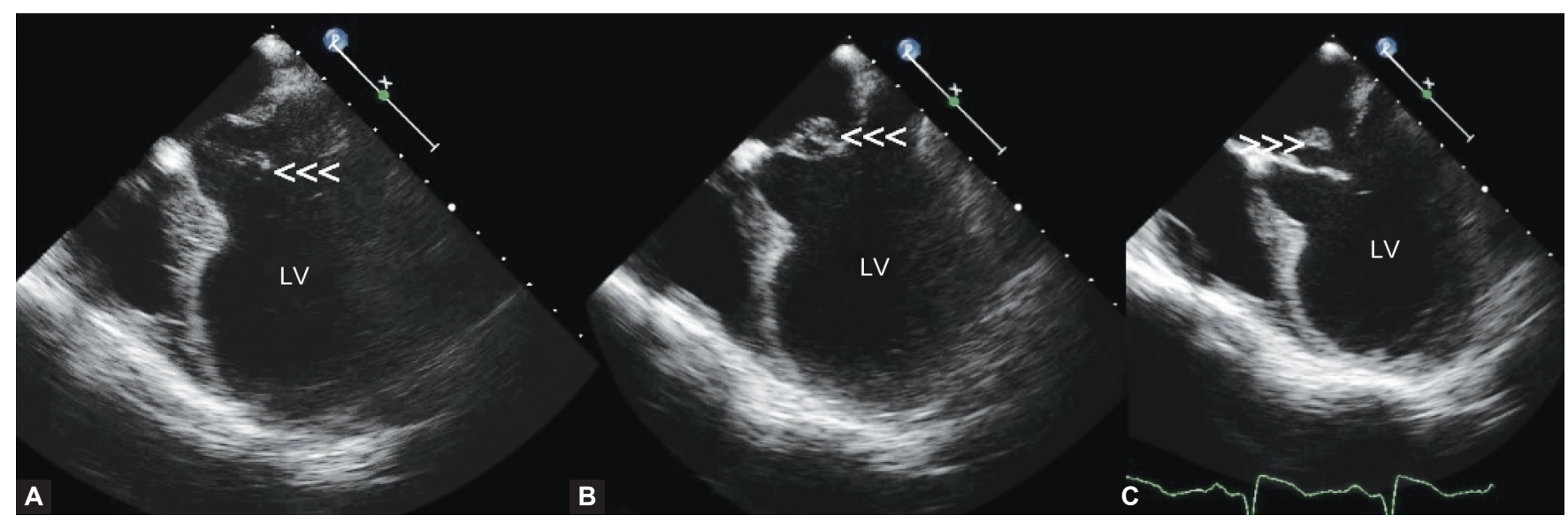

Figs $1 \mathrm{~A}$ to $\mathrm{C}$ : Perioperative transesophageal echocardiography showing an echogenic intracardiac mass (arrow heads) measuring $18 \mathrm{~mm}$ in length in a patient with postinfarction severe mitral regurgitation (LV: left ventricle)

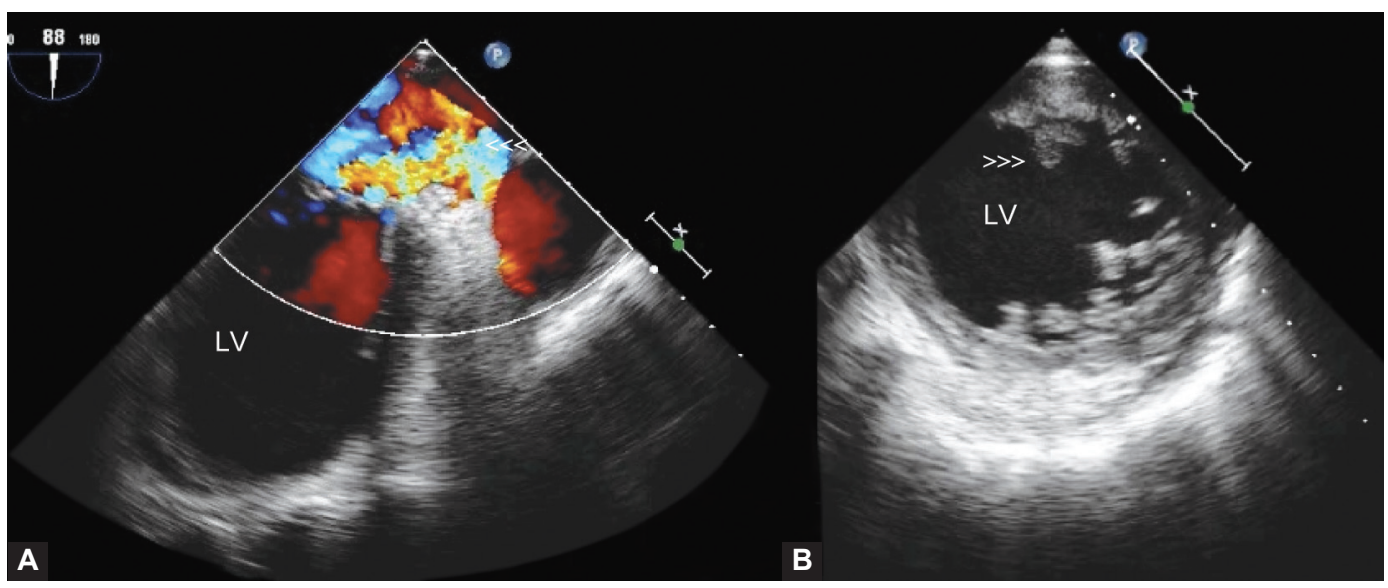

Figs 2A and B: (A) Color Doppler perioperative transesophageal echocardiography showing eccentric anteromedial jet of mitral regurgitation in a patient with postinfarct severe mitral regurgitation. (B) Transgastric view demonstrating single intact anterolateral papillary muscle and two heads of posteriomedial papillary muscle with cut-off sign suggestive of partial rupture of one of the heads of PM (LV: left ventricle) 
muscles send chordae tendineae to both leaflets of the mitral valve; as a result, either leaflet can be affected by rupture of either PM.

In this patient initially, there was no documentation of MR. After a week, he presented with severe MR and a mobile mass prolapsing predominantly in left atrium just beneath the anterior mitral leaflet and PML prolapse. There was no evidence of chordae rupture (flail MV leaflet or direct visualization) and the PM looked to be intact on transthoracic echocardiography. However, perioperative TEE detected severe akinesia of all the segments with hyper contractile lateral wall along with two heads of posteriomedial PM with cut-off sign suggestive of partial PM head rupture, which was confirmed on surgical inspection. Thus, in this patient, acute ischemic MR was ruled out and the patient satisfied the criteria for chronic ischemic MR, namely, presenting after 1 week, near global akinetic segments, significant coronary artery disease, and structurally normal MV leaflets and chordae on transesophageal echocardiogram. However, in addition to mechanism for chronic MR, this patient had partial rupture of one of the heads of posteriomedial PM. This is explained by Calvo et al. ${ }^{7}$ They have postulated that the preserved contractility or hyper contractile adjacent myocardium exerts increased stress on an already compromised PM, eventually leading to rupture as noted in this patient. In conclusion, this case illustrates a rare presentation of postinfarct rupture of one of the heads of posteriomedial PM occurring late after infarction presenting as an intracardiac mass. This has to be considered in such patients as this may have surgical implications.

\section{REFERENCES}

1. Borger MA, Alam A, Murphy PM, Doenst T, David TE. Chronic ischemic mitral regurgitation: repair, replace or rethink? Ann Thorac Surg 2006;81:1153-1161.

2. Agricola E, Oppizzi M, Pisani M, Meris A, Maisano F, Margonato A. Ischemic mitral regurgitation: mechanisms and echocardiographic classification. Eur J Echocardiogr 2008; 9:207-221.

3. Piérard LA, Carabello BA. Ischaemic mitral regurgitation: pathophysiology, outcomes and the conundrum of treatment. Eur Heart J 2010;31:2996-3005.

4. Moursi MH, Bhatnagar SK, Vilacosta I, et al. Transesophageal echocardiographic assessment of papillary muscle rupture. Circulation 1996;94:1003-1009.

5. Kerut EK, Hanawalt C, Everson C. Echo features of posteromedial papillary muscle rupture without papillary muscle prolapse into the left atrium. Echocardiography 2011;28: 1046-1048.

6. Tempe DK, Datt V, Banerjee A, Gandhi A. Chordae tendineae from posteromedial papillary muscle inserting into the anterior mitral leaflet: an unusual presentation. Ann Card Anaesth 2007;10:63.

7. Calvo FE, Figueras J, Cortadellas J, Soler-Soler J. Severe mitral regurgitation complicating acute myocardial infarction: clinical and angiographic differences between patients with and without papillary muscle rupture. Eur Heart J 1997;18:1606-1610. 